\title{
Evaluación de un método no destructivo para estimar las concentraciones de clorofila en hojas de variedades de uva de mesa
}

\author{
Evaluation of a non-destructive method to estimate the concentration \\ of chlorophyll in leaves of table grape $\mathrm{cv}$.
}

\author{
Rodrigo Callejas $^{1 *}$, Erika Kania $^{1}$, Américo Contreras ${ }^{1}$, Cecilia Peppi $^{1}$ y Luis Morales ${ }^{2}$
}

\section{RESUMEN}

Durante la temporada 2009-2010 se llevaron a cabo calibraciones del Medidor Portátil de Clorofila (Modelo CCM-200, Opti Sciences, USA), en Vitis vinifera, variedades Red Globe y Thompson Seedles. De manera adicional se procedió a comparar este último equipo con el medidor SPAD 502 (Konica Minolta, Osaka, Japan) sobre vides variedad Flame seedles. Los resultados del medidor portátil CCM-200, expresado en unidades CCI, obtenidas mediante lectura de reflectancia de las hojas a ciertas longitudes de onda fueron comparadas mediante un análisis de regresión con la extracción de clorofila y posterior lectura espectrofotométrica. Los análisis de regresión, para la variedad Thompson Seedless, mostraron un alto coeficiente de determinación $\left(r^{2}=0,99\right)$, expresándose esta en una ecuación lineal de segundo orden; mientras que para la variedad Red Globe se observó un alto coeficiente de determinación $\left(r^{2}=0,93\right)$, ajustados también a un polinomio de segundo orden. La comparación entre ambos equipos (Flame Seedless) arrojó un coeficiente de determinación de $r^{2}=0,95$, ajustado también a un polinomio de segundo orden. Para una temporada 2010, la evolución del contenido de clorofila en las variedades Flame Seedless y Thompson Seedless fue medida usando el equipo CCM200. Para ambas variedades, los mayores contenidos se observaron al final de pinta, aunque el mejor momento para estimar el contenido de clorofila corresponde al comienzo de floración. La variabilidad de las mediciones, en diferentes momentos del día y en diferentes posiciones de la hoja en el brote (basal, medio y apical) fue evaluado en Thompson Seedles, siendo significativas solo para la posición de la hoja en el brote. Los resultados sugieren que para calibraciones realizadas sobre distintas variedades y ubicaciones, la utilización de medidores portátiles de clorofila son una alternativa para el monitoreo de clorofila en vides.

Palabras clave: medidor portátil de clorofila, CCM-200, SPAD-502, Vitis vinifera L.

\begin{abstract}
During 2009 and 2010, calibration of the Portable Chlorophyll Meter (Model CCM-200, Opti Sciences, USA) was carried out using Vitis vinifera L. cvs. Red Globe and Thompson Seedless. Aditionally, on cv. Flame Seedless, the Chlorophyll Meter was compared with the SPAD 502 meter (Konica Minolta, Osaka, Japan). The results of the portable meter CCM-200 were compared, using regression analysis, with chlorophyll extraction and subsequent spectrophotometric reading. Regression analysis for Thompson Seedless showed a high coefficient of determination $\left(r^{2}=0.99\right)$ and data adjusted to a second order linear equation; while for Red Globe there was a high coefficient of determination $\left(r^{2}=0.93\right)$, with data adjusted to a second order polynomial. The comparison between the two portable meters (Flame Seedless) showed a coefficient of determination $r^{2}=0.95$ adjusted to a second order polynomial. Also, to 2010 seasons, the chlorophyll content evolution of 'Flame Seedless' and 'Thompson Seedless' was measured using the CCM-200 instrument. For both cvs. the highest chlorophyll content was observed close to veraison, however the optimum time to estimate chlorophyll content would be at the beginning of bloom. Variability of measurements at different times of the day and on different positions in the shoot (basal, middle, apical) was evaluated in 'Thompson Seedless' cv, being significant only the leaf shoot position. Results suggest that, previous calibration according to cultivar and location, portable chlorophyll meters are a suitable alternative to monitor chlorophyll content.
\end{abstract}

Key words: portable chorophyll meters CCM-200, SPAD-502, Vitis vinifera $L$.

1 Universidad de Chile, Facultad de Ciencias Agronómicas. Casilla 1004, Santiago.

2 Universidad de Chile, Facultad de Ciencias Agronómicas. Departamento de Ciencias Ambientales y Recursos Naturales Renovables. Laboratorio de Investigación en Ciencias Ambientales LARES.

* Corresponding author. E-mail address: rcalleja@uchile.cl

Fecha de Recepción: 27 Junio, 2012.

Fecha de Aceptación: 26 Agosto, 2013. 


\section{Introducción}

La producción de uva de mesa en la Región de Atacama, Chile, está sujeta a importantes dificultades edafoclimáticas que obligan a realizar todos los esfuerzos técnicos posibles que permitan evaluar y controlar condiciones adversas de crecimiento y desarrollo de la fruta. Este clima se caracteriza por una alta radiación solar y altas temperaturas máximas diarias. Las condiciones generales de los suelos en esta área son: valores de $\mathrm{pH}$ entre 7,8-8,5, conductividad eléctrica (CE) entre 1,2 a $4 \mathrm{dS} \cdot \mathrm{m}^{-1}$, altos niveles de sodio, cloro y boro (los que generan problemas de toxicidad en las plantas) y suelos de texturas gruesas con bajos niveles de microelementos. Estas condiciones adversas requieren la implementación de nuevas tecnologías que permitan un control más efectivo del comportamiento fisiológico de las plantas, con el fin de optimizar los manejos agronómicos en esta zona.

Una de las variables que se deben tomar en consideración para determinar el estatus fisiológico de las plantas en un momento determinado es el contenido de clorofila, responsable de la coloración verde de las hojas (Steele et al., 2008). Esto se da principalmente por la respuesta que pueden tener las plantas a modificar su concentración interna en fenómenos como exceso de salinidad (Sivritepe, 1999; Alizadeth et al., 2010), estrés lumínico, estrés hídrico (Flexas et al., 1999), daños causado por ozono (Neufeld et al., 2006) e incluso daño causados por insectos (Banchfield et al., 2006), siendo en definitiva esta variación una respuesta a cualquier condición medioambiental, fitosanitario o de fertilización que afecte al propio metabolismo de la hoja (Orihuela et al., 2007). Junto a esto, el contenido de clorofila se encuentra altamente asociado a las concentraciones de nitrógeno en las hojas, lo que ha sido evaluado mediante los cambios en las particiones de nitrógeno hacia la síntesis de clorofila en plantas sometidas a baja luminosidad, lo que hace la medición de clorofila una estimación indirecta de nitrógeno (Evans, 1989).

La utilización de medidores portátiles de clorofila están basados principalmente en mediciones de absorbancia de dos diferentes anchos de banda, las que varían entre el rojo y el rojo lejano. La luz roja es intensamente absorbida por la clorofila y la fracción correspondiente al rojo lejano es utilizada como una longitud de onda de referencia (Markwell, 1995), siendo ambas lecturas utilizadas para crear un índice de clorofila, altamente correlacionado a un valor relativo de clorofila total.

Los equipos medidores de clorofila SPAD502 y el CCM-200 presentan similitudes en los fundamentos de uso, pero difieren en los anchos de banda utilizados. Es así como el SPAD 502 utiliza dos longitudes de onda central de $650 \mathrm{~nm}$ (rojo) y $940 \mathrm{~nm}$ (infrarrojo) (Kapotis et al., 2003) y el CCM-200 con longitudes de 665 (rojo) y $940 \mathrm{~nm}$ (infrarrojo), respectivamente (Cate y Perkins, 2003). La estimación de clorofila en ambas metodologías se basa en la diferencia en la medición de transmitancia a través de la hoja evaluada. Ambos equipos, mediante la utilización de dos diodos, emiten por medio de la hoja 2 anchos de banda (rojo e infrarrojo), los que son posteriormente captados por fotodiodos ubicados posteriores a la hoja. El principio de ambos medidores de clorofila se basa en la considerable absorción de luz roja por parte de la clorofila, no así el infrarrojo, siendo la lectura de ambas longitudes llevadas a un valor índice (unidades SPAD o CCI), permitiendo una medición indirecta mediante la correlación de ambos índices con el contenido de clorofila de las hojas (Chang and Robinson, 2003).

Debido a las ventajas de conocer los contenidos de clorofila en hojas de vid en zonas áridas, el uso de medidores portátiles de clorofila se perfila como una oportunidad tecnológica, rentable, económica y factible de ser utilizada en la medida que sean calibrados para cada variedad.

Los objetivos de esta investigación fueron: comparar las mediciones entre el medidor portátil de clorofila SPAD-502 y el CCM-200, calibrar el medidor portátil de clorofila CCM-200 para dos variedades de uva de mesa; determinar la variación del contenido de clorofila a lo largo de la temporada y verificar la variabilidad de las mediciones a lo largo del brote durante el día.

\section{Materiales y Métodos}

El estudio se realizó entre agosto del 2009 y octubre de 2010, en dos huertos de uva de mesa ubicados en el valle del Huasco, comuna de Vallenar, Región de Atacama, Chile (28 $54^{\prime} 52.60^{\prime \prime}$ S 70¹6' 35.50 " O, altitud $1117 \mathrm{~m}$ ), y en el valle de Copiapó, sector de Los Loros, comuna de Tierra Amarilla, Región de Atacama, Chile (27\%49'50.47' S 7006' 59.59" O, altitud $937 \mathrm{~m}$ ), en tres variedades de uva de mesa (Red Globe, Thompson Seedless y Flame Seedless). 


\section{Comparación de equipos}

Se procedió a elegir 50 muestras de hojas de vid madura, variedad Flame Sedles, en plantas ubicadas en el valle del Huasco, con diferente tonalidad, para verificar el comportamiento entre el SPAD 502 (Konica Minolta, Osaka, Japan) y el CCM200 (Opti Sciences, Tynsgboro, Massachussets, USA). Cada hoja fue evaluada en el mismo lugar con ambos equipos.

\section{Calibración CCM-200}

Posterior a la comparación de ambos métodos se procedió a seleccionar 30 hojas de diversas tonalidades de la variedad Red Globe y Thompson Seedless en plantas ubicadas en el valle de Copiapó para realizar, mediante la utilización del equipo portátil CCM-200, la calibración de sus mediciones. Se decidió realizar las evaluaciones en 2 variedades, para verificar si existen diferencias entre las calibraciones, porque las mediciones en unidades CCI pueden verse afectadas principalmente por especies, género, diferencias dentro de especies y condiciones de crecimiento (Pinkard et al., 2006). Las evaluaciones se realizaron en el tercio inferior de la hoja, procediendo a recortar el círculo de la hoja evaluada, incorporándola inmediatamente en nitrógeno líquido y enviándolas posteriormente al laboratorio para el análisis de clorofila total. Para esto se maceró las muestras en un mortero a $-20^{\circ} \mathrm{C}$ sobre hielo, con $500 \mu \mathrm{l}$ de etanol al $96 \%$, en completa oscuridad. Una vez molida la muestra fue transferida a un tubo eppendorf, agregándole $500 \mu \mathrm{l}$ de etanol al $96 \%$ sobre el mortero y luego centrifugado por 5 minutos a $2500 \mathrm{G}$ y $4{ }^{\circ} \mathrm{C}$. Posteriormente el contenido de pigmentos se determinó por medio de un espectrofotómetro de doble beam, leyendo la absorbancia a 665, 649 y $470 \mathrm{~nm}$. El cálculo del contenido de pigmentos se detalla en las ecuaciones de la Tabla 1.

Los valores de clorofila obtenidos en laboratorio fueron analizados mediante regresiones lineales, con el fin de obtener curvas ajustadas para cada variedad, de acuerdo con los valores obtenidos con las mediciones del CCM- 200.

Para determinar la ecuación que presenta la mejor capacidad de ajuste, tanto entre las mediciones de los dos equipos como para la calibración entre el CCM-200 y las dos variedades de vid, se procedió a obtener el error cuadrático medio de predicción (Hamilton, 1994).
Tabla 1. Fórmulas utilizadas para el cálculo de las clorofilas (Lichtenthaler, 1987; citado por Blackburn, 1998).

\begin{tabular}{ll}
\hline Clorofila $a\left(\mu \mathrm{g} / \mathrm{cm}^{2}\right)$ & $=13,96 \mathrm{~A}_{665}-6,88 \mathrm{~A}_{649}$ \\
Clorofila $b\left(\mu \mathrm{g} / \mathrm{cm}^{2}\right)$ & $=24,96 \mathrm{~A}_{649}-7,32 \mathrm{~A}_{665}$ \\
Carotenoides totales $\left(\mu \mathrm{g} / \mathrm{cm}^{2}\right)=$ & $\left(1000 \mathrm{~A}_{470}-2,05\right.$ Clorofila \\
& $a-114,8$ Clorofila $b) / 245$
\end{tabular}

\section{Variación en las mediciones a lo largo de la temporada}

Para determinar la variación del contenido de clorofila durante la estación de crecimiento fueron monitoreadas una planta de la variedad Flame Seedless y otra de la variedad Thompson Seedless. Ambas plantas fueron evaluadas de viñas provenientes de un sector con historial de buenas producciones y calidad de fruta, buen desarrollo de follaje y sin aplicaciones de fertilizantes foliares hasta plena flor.

En dos brotes por planta, marcados en el sector medio del cargador, se evaluó semanalmente todas las hojas del brote, desde que el brote midió $20 \mathrm{~cm}$ hasta inicio de cuaja, usando el equipo portátil CCM-200 (Unidades CCI), manteniendo la individualidad de cada hoja. La hoja opuesta al racimo se consideró como 0, las hojas basales correspondieron a las posiciones negativas y las posiciones positivas a las hojas distales.

\section{Variación en las mediciones a lo largo del día}

Para verificar si existen diferencias entre mediciones realizadas a distintas horas del día y entre la posición de la hoja a lo largo del brote, se procedió a evaluar 4 brotes en una planta, variedad Thompsos Seedless, realizando evaluaciones del sector basal, medio y apical del brote en 3 momentos del día (8:00 AM; 12:00 PM y 16:00 PM). Se utilizó un diseño completamente aleatorizado con estructura factorial de los tratamientos, con el fin de determinar si existe interacción entre el factor "ubicación de la hoja en el brote" (basal, medio y apical) y el factor "hora del día" (8:00 AM; 12:00 PM y 16:00 PM). La unidad experimental correspondió a la hoja. En las mediciones de las hojas se tomaron 5 puntos por lámina, para obtener una estimación de mayor confiabilidad, siendo realizada a inicio del período de floración (30 de octubre de 2010). 


\section{Resultados}

\section{Comparación de equipos}

La Figura 1 muestra el ajuste obtenido en la medición de las lecturas con el SPAD-502 y el CCM-200 en la variedad Flame Seedless. Se observó un mayor ajuste entre las observaciones que presentaron las menores lecturas de los equipos. $\mathrm{La}$ curva que mayor ajuste generó fue un polinomio de segundo orden $\left(\mathrm{n}=40 ; r^{2}=0,95 ; p\right.$-value $\left.\leq 0,0001\right)$, determinado por la ecuación CCI: 4,44-0,10SPAD $+0,02 \mathrm{SPAD}^{2}$.

\section{Calibración del medidor portátil CCM-200}

Para la variedad Thompson Seedless (Figura 2) se observa una acentuada relación lineal entre ambas variables, expresándose en un polinomio de segundo orden (clorofila total $\left(\mu \mathrm{m} \cdot \mathrm{cm}^{-2}\right)=56,8+$ $16,8 \mathrm{CCI}+0,1326 \mathrm{CCI}^{2} ; \mathrm{n}=15 ; r^{2}=0,99 ; p$-value $\leq 0,0001)$. Este ajuste se repite en la variedad Red Globe (Figura 3), cuyo mejor ajuste se realizó mediante una regresión lineal, expresándose también en un polinomio de segundo orden (clorofila total $\left(\mu \mathrm{m} \cdot \mathrm{cm}^{-2}\right)=82,134+12,1 \mathrm{CCI}-0,08 \mathrm{CCI}^{2} ; n=15$ $r^{2}=0,93 ; p$-value $\left.<0,0001\right)$.

\section{Variación en las mediciones a lo largo de la temporada}

En las Figuras 4 y 5 se puede apreciar la evolución del contenido de clorofila dependiendo de la posición de la lámina en el brote, así como de la fecha de evaluación para la variedad Flame Seedles y Thompson Seedles, respectivamente.

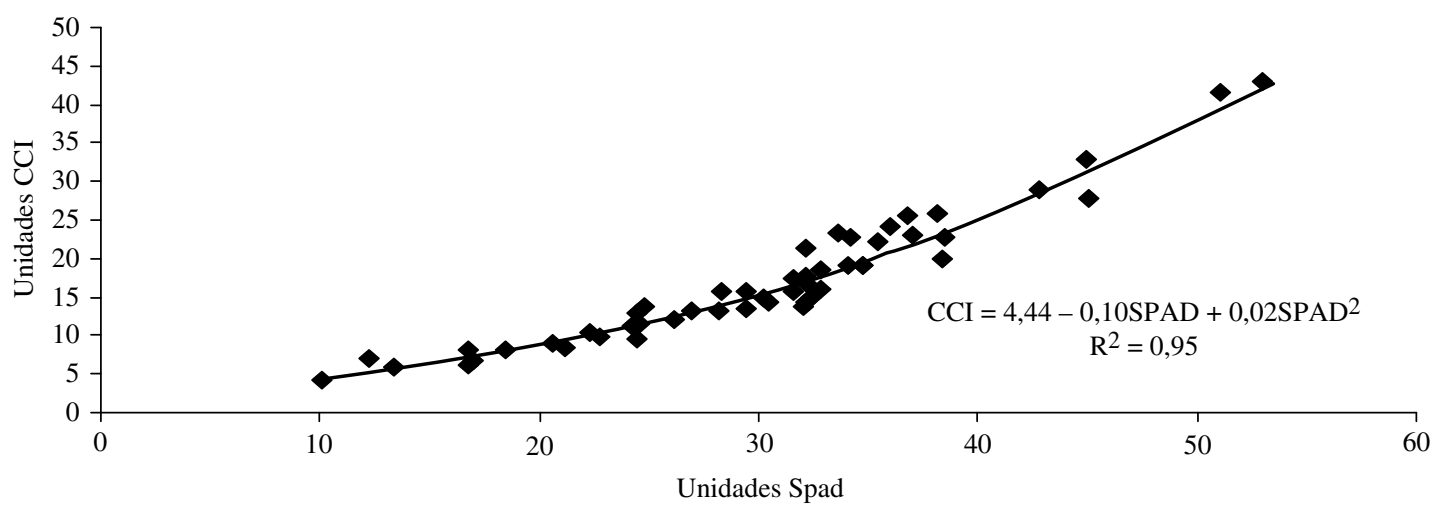

Figura 1. Relación entre las lecturas obtenidas mediante el SPAD-502 (Unidades SPAD) y el CCM-200 (Unidades CCI); evaluadas en la variedad Flame Seedless.

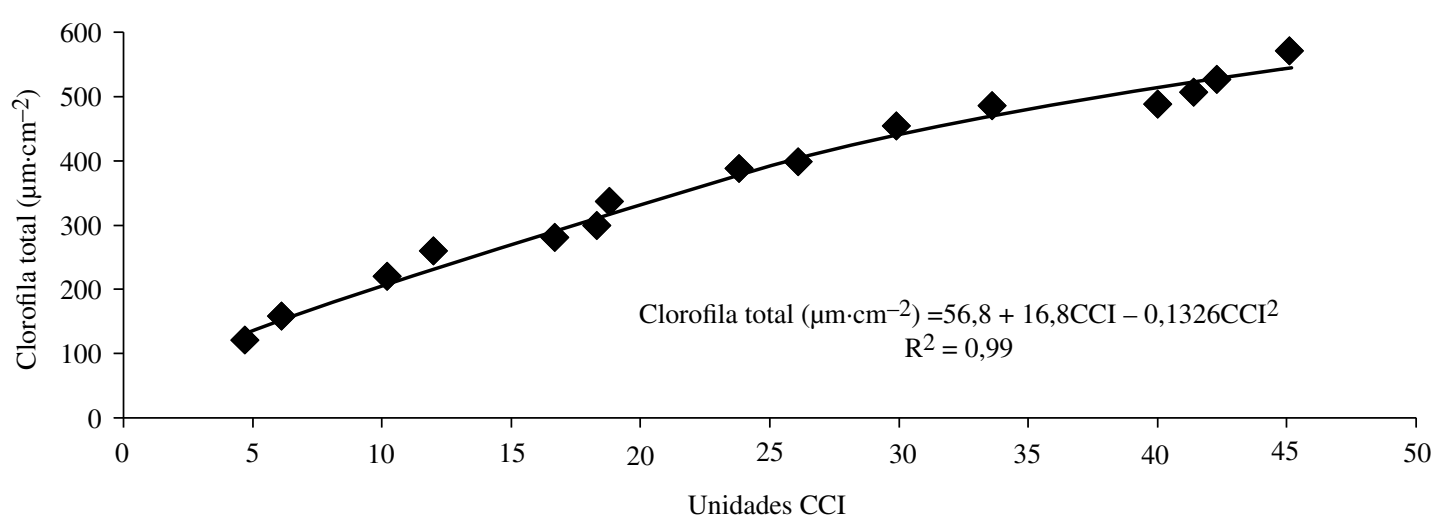

Figura 2. Relación entre las lecturas obtenidas mediante el equipo Opti-Science CCM-200 (Unidades CCI) y el contenido de clorofila total $\left(\mu \mathrm{m} \cdot \mathrm{cm}^{-2}\right)$, en la variedad Thompson Seedless. 


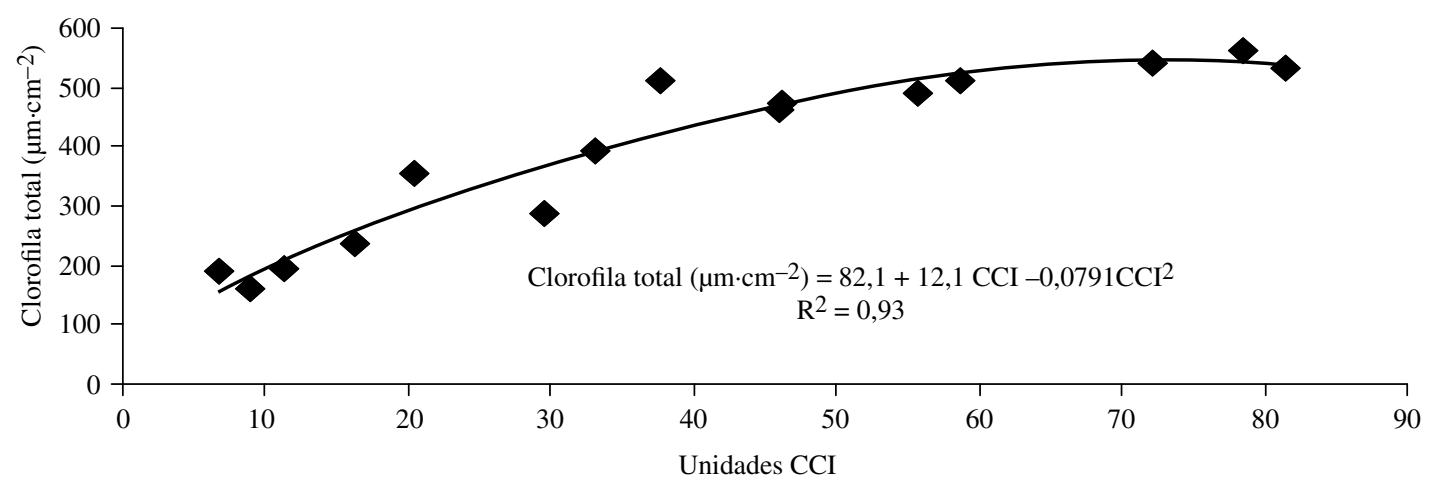

Figura 3. Relación entre las lecturas obtenidas mediante el equipo Opti-Science CCM-200 (Unidades CCI) y el contenido de clorofila total $\left(\mu \mathrm{m} \cdot \mathrm{cm}^{-2}\right)$, en la variedad Red Globe.

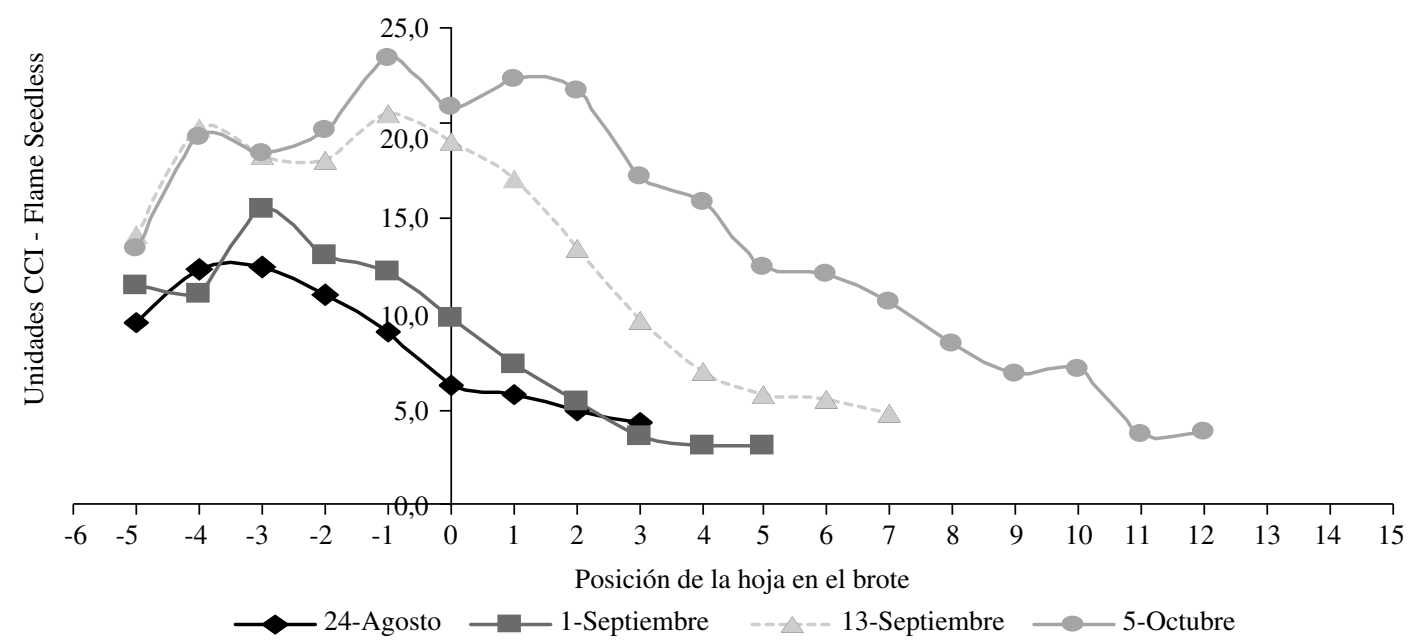

Figura 4. Tendencia en la variación de las unidades CCI en la variedad Flame Seedless de acuerdo con la fecha de evaluación y posición de la hoja en el brote (inicio brotación: 20 julio; plena flor: 13 septiembre).

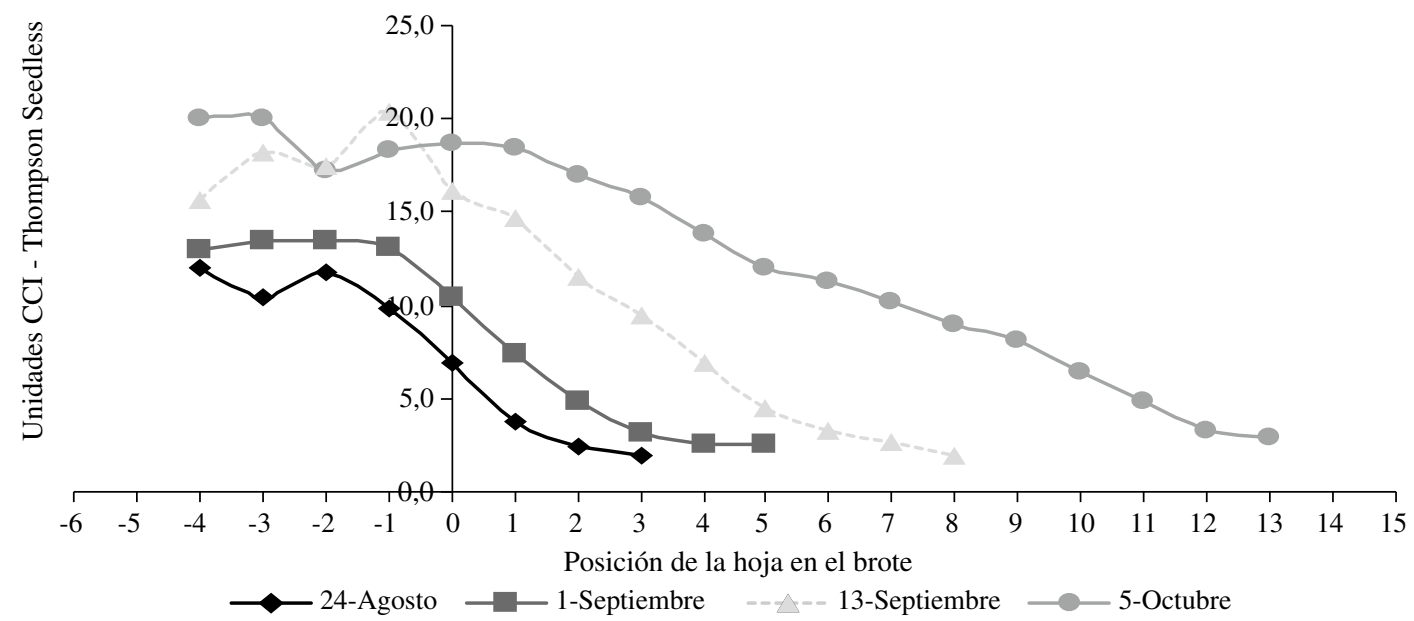

Figura 5. Tendencia en la variación de las unidades CCI en la variedad Thompson Seedless, de acuerdo con la fecha de evaluación y posición de la hoja en el brote (inicio brotación: 25 julio; plena flor: 18 septiembre). 
Para ambas variedades, en general, el contenido de clorofila decreció durante la temporada y las lecturas fueron menores en las hojas distales.

\section{Variación en las mediciones a lo largo del día}

Las evaluaciones en tres momentos del día no arrojaron diferencias significativas entre la interacción de los factores "ubicación de la hoja en el brote" y "hora del día" ni en el factor "hora del día". Para el caso del factor "ubicación de la hoja en el brote" se observaron diferencias significativas (Figura 6), demostrando que la ubicación de las hojas en el sector de la base, medio y apical de los brotes presentan diferentes valores al momento de realizar evaluaciones con el CCM-200.

\section{Discusión}

\section{Comparación de equipos}

Curvas similares a las encontradas en la calibración de equipos CCM y Spad 502 (Figura 1) fueron determinadas por Richardson et al. (2002), quienes encontraron un mejor ajuste mediante un polinomio de tercer orden: (CCI: 0,964 +0,185SPAD $\left.-0,002 \mathrm{SPAD}^{2}+0,0002 \mathrm{SPAD}^{3}\right)$ con un $r^{2}=0,97$. Las disparidades podrían estar asociadas a las diferencias de lectura entre ambos equipos, principalmente a la variedad de longitudes de ondas emitidas y las diversas áreas que estos equipos evalúan. Es así como el SPAD-502 presenta un área de lectura de
$0,06 \mathrm{~cm}^{2}$ y el CCM-200 $0,71 \mathrm{~cm}^{2}$, presentando este último una mayor superficie de muestreo. Lo anterior no asegura una mayor confiabilidad en el CCM-200 respecto del SPAD, ya que en mediciones en hojas de abedul la precisión del SPAD-502 fue superior a CCM-200, a pesar de tener un área de medición diez veces menor que este último (Richardson et al. 2002).

Chang y Robinson (2003) sugieren que esta limitación puede ser corregida realizando un mayor muestreo por hoja por parte del equipo portátil, adquiriendo un valor más representativo por cada unidad de muestreo, esto con el objetivo de ser capaz de generar un índice de mayor confiabilidad, controlando la variabilidad dentro de la muestra y creando a su vez medidas más ajustadas.

\section{Calibración del medidor portátil CCM-200}

A pesar de los ajustes similares encontrados para ambas variedades, es necesario destacar que las mediciones con equipos portátiles requieren de calibraciones particulares para cada especie e incluso variedades, esto con el fin de ajustar de manera más precisa las estimaciones de clorofila (Loh et al., 2002). En mediciones de equipos portátiles de clorofila en especies del género Vitis, Steele et al. (2008) reportaron para Vitis lambrusca (Minnesota $78 \mathrm{x}$ Ontario) un modelo ajustado a una regresión lineal de segundo grado, consistente con lo encontrado en este estudio, pero advirtiendo que entre valores de 300 y $576 \mu \mathrm{m} \cdot \mathrm{cm}^{-2}$ el modelo respondía solo

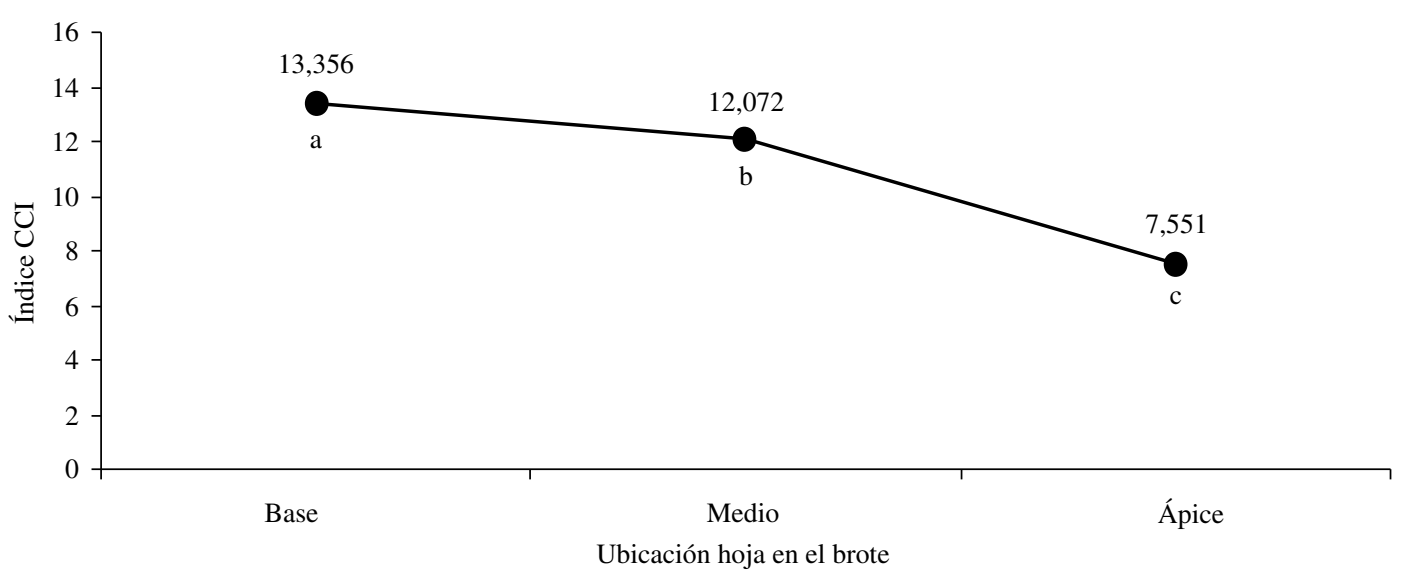

Figura 6. Variación entre las mediciones de las unidades CCI en tres posiciones a lo largo del brote en variedad Thompson Seedless. Letras distintas indican diferencias significativas entre las tres posiciones a lo largo del brote, según test de comparación múltiple de Tukey ( $\mathrm{p}$ value $\leq 0,0001)$. 
en el $45 \%$, por lo que sugiere un mayor uso en etapas tempranas de desarrollo, debido a su escaso ajuste a valores mayores de $300 \mu \mathrm{m} \cdot \mathrm{cm}^{-2}$. Hawkins et al. (2008) reportaron escaso ajuste a mayores concentraciones de clorofila en Lindera melissifolia, y Richardson et al. (2002) encontraron tanto para mediciones realizadas con SPAD-502 como CCM200 mayor dispersión en altos valores de clorofila, lo que sugiere atender esta información al realizar calibraciones de equipos portátiles, a pesar de no encontrarse esta variabilidad en el presente ensayo.

\section{Variación en las mediciones a lo largo de la temporada}

La elección de las hojas al realizar las mediciones toma vital trascendencia, debido a que la lectura de los medidores portátiles varía notablemente entre hojas apicales de vid y hojas completamente extendidas, y entre plantas sometidas a estrés hídrico y plantas bajo riego (Fanizza et al., 1991), por lo tanto la estandarización de las hojas al realizar el muestreo resulta de gran importancia. Curvas similares a las encontradas en este estudio, pero en $V$. vinifera $\mathrm{cv}$. White Riesling, fueron descritas por Zulini et al. (2007), observándose una disminución de clorofila (medida como unidades SPAD) a medida que las hojas se acercan al ápice. Las hojas más basales presentaron los mayores índices de CCI a una misma fecha de evaluación, aumentando el contenido de clorofila hasta la evaluación de octubre. Para ambas variedades, el incremento de la concentración de clorofila en las hojas de la base (bajo la inserción del racimo) se estabiliza aproximadamente a los 50 días de brotación (Figuras 4 y 5). Estos resultados concuerdan con lo encontrado con Kriedemann (1968), quien indica que el contenido de clorofila, el área foliar y la absorción de la radiación incidente aumentan con la edad de la hoja. A su vez, la tendencia en el comportamiento del contenido de clorofila son semejantes a los encontrados por Candolfi-Vasconcelos y Koblet (1991) y Bertamini y Nedunchezhian (2003), quienes observaron un aumento gradual del contenido de clorofila, alcanzando un máximo nivel hasta envero, para luego disminuir progresivamente hacia cosecha, siendo probablemente esta disminución un mecanismo de adaptación de las hojas a la luz o bien a una senescencia temprana.

Según los antecedentes aportados por Steele $\mathrm{et} \mathrm{al}$. (2008), se podría estimar que la mayor dispersión asociada con los mayores niveles de clorofila se daría de manera tardía en la temporada, por lo que es recomendable que las mediciones de clorofila se realicen de manera temprana en la temporada, lo que entregaría un buen indicador del estatus fisiológico al inicio de la temporada.

\section{Variación en las mediciones a lo largo del día}

Los equipos portátiles basados en mediciones indirectas de clorofila mediante la transmitancia de luz dependen no solo de la cantidad de clorofila, sino además de su distribución dentro de la hoja, la que está relacionada directamente con el arreglo de los cloroplastos dentro de la hoja. En condiciones de laboratorio, plantas de tabaco sometidas a luz azul (340 $\mu \mathrm{mol}$ de fotones $\mathrm{m}^{-2} \mathrm{~s}^{-1}$ ) presentaron un reordenamiento de los cloroplastos, lo que influyó en las mediciones realizadas posteriormente con el medidor de clorofila SPAD-502, presentando los menores valores SPAD las plantas sometidas a radiación directa de luz azul (Nauš et al., 2010). Movimiento de cloroplastos mediante la radiación de luz azul también fueron detectadas en helechos Adiantum prothallus, los cuales posterior al cese de la luz retomaron su posición inicial (Wada et al., 2003). Es probable que para las condiciones de desarrollo de las plantas de vid en Atacama la proporción de radiación de luz azul no varíe a lo largo del día, lo que genera mediciones homogéneas, independiente del momento en que se realice.

\section{Conclusiones}

Las mediciones mediante equipos portátiles de clorofila entregan una medición rápida y confiable del contenido total de clorofila en hojas de $V$. vinifera var. Thompson Seedless y Red Globe, demostrando que deben realizarse calibraciones para cada variedad en particular. Además, es importante la elección del momento en la temporada y del sector en los cuales se realizarán las mediciones, principalmente por la alta variabilidad en los contenidos de clorofila que se presentan en los brotes, aconsejándose realizarlo en las hojas del sector basal del brote (bajo la inserción del racimo) a partir de plena flor. Tanto el SPAD-502 como el CCM-200 presentaron una regresión lineal de segundo grado, explicada probablemente por las diferencias entre las longitudes de onda empleadas y las superficies de lecturas de ambos equipos. 


\section{Literatura Citada}

Alizadeht, M., S. Singh, V. Patel, R. Bhattacharya, and B. Yadav. 2010. In vitro responses of grape rootstocks to $\mathrm{NaCl}$. Biologia Plantarum 54 (2): 381-385.

Banchieldf, A.S. Robinson, L. Renzullo, and K. Powell. 2006. Can leaf pigment composition help us identify grapevines infested with phylloxera? Functional Plant Biology 33: 507-517.

Blackburn, G.

1998. Spectral indices for estimating photosynthetic pigment concentrations: a test using senescent tree leaves. Int. j. Remote Sensing 19 (4). 657-675.

Bertamini, M., and N. Nedunchezhian.

2003. Photosynthetic functioning of individual grapevines leaves (Vitis vinifera L. cv Pinot noir) during ontogeny in the field. Vitis 42 (1) 13-17.

Candolfi-Vascocelos, M., and W. Koblet.

1991. Influence of partial defoliation on gas exchange parameters and chlorophyll content on - field grown - grapevines mechanisms and limitations of the compensation capacity. Vitis 30: 129-141.

Cate, T., and T. Perkins.

2003. Chlorophyll content monitoring in sugar maple (Acer saccharum). Tree Physiology 23: 1077-1079.

Chang, S., and D. Robinson

2003. Nondestructive and rapid estimation of hardwood foliar nitrogen status using the SPAD-502 chlorophyll meter. Forest Ecology and Management 181: 331-338.

Evans, J.

1989. Photosynthesis and nitrogen relationships in leaves of $\mathrm{C}_{3}$ plants. Oecologia 78: 9-19.

Fanizza, G., L. Ricciardi, and C. Bagnulo.

1991. Leaf greenness measurements to evaluate water stressed genotypes in Vitis vinifera. Euphytica 55: 27-31.

Flexas, J.J. Escalona, and H. Medrano.

1999. Water stress induces different levels of photosynthesis and electron transport rate regulation in grapevines. Plant, Cell and Environment 22: 39-48.

Hamilton, J.D.

1994. Time Series Analysis. Princeton, New Jersey: Princeton, Univ. Press.

Hawkins, T., E. Gardiner, and G. Comer.

2008. Modeling the relationship between extractable chlorophyll and SPAD-502 readings for endangered plant species research. Journal for Nature Conservation 17: 123-127.
Kapotis, G., G. Zervoudakis, T. Veltsistas, and G. Salahas.

2003. Comparaison of chlorophyll meter readings with leaf chlorophyll concentration in Amaranthus vlitus: correlation with physiological processes. Russian Journal of Plant Physiology 50 (3): 395-397.

Kriedemann, P.

1968. Photosynthesis in vine leaves as function of light intensity, temperature, and leaf age. Vitis 7: 213-220.

Loh, F., J. Grabosky, and N. Bassuk.

2002. Using the Spad 502 meter to assess chlorophyll and nitrogen content of benjamin fig and cottonwood leaves. HortTechnology 12 (4) 682-686.

Nauš, J., J. Prokopová, and J. Řebíček.

2010. Spad chlorophyll meter reading can be pronouncedly affected by chloroplast movement. Photosynthesis Research. 105 (3) 265-271.

Neufled, H., A. Chappelka, G. Somers, K Burkey, A. Davison, and P. Finkelstein.

2006. Visible foliar injury caused by ozone alters the relationships between SPAD meter reading and chlorophyll concentration in cutleaf coneflower. Photosynthesis Research 87 (3): 281-286.

Orihuela, D., J. Hernández, R. Maestre, y R. López-Bellido.

2007. Calibración de un minolta Spad meter en un cultivo de fresón. Actas de Horticultura 48: 340-343.

Pinkard, E., V. Patel, and C. Mohammed.

2006. Chlorophyll and nitrogen determination for plantationgrown Eucalyptus nitens and E. globulus using a non-destructive meter. Forest Ecology and Management 223: 211-217.

Richardson, A., S. Duigan, and G. Berlyn.

2002. An evaluation of noninvasive methods to estimate foliar chlorophyll content. New Phytologist 153: 185-194.

Sivritepe, N., and A. Eriş.

1999. Determination of salt tolerance in some grapevine cultivars (Vitis vinifera L.) under in vitro conditions. Tr. J. Of Biology 23: 473-485.

Steele, M., A. Gitelson, and D. Rundquist.

2008. A comparaison of two techniques for nondestructive measurement of chlorophyll content in grapevine leaves. Agronomy Journal 100 (3): 779-782.

Wada, M., T. Kagawa, and Y. Sato.

2003. Chloroplast movement. Annu. Rev. Plant Biol. 455-468.

Zulini, L., M. Rubinigg, R. Zorer, and M. Bertamini.

2007. Effects of drought stress on chlorophyll fluorescente and photosynthetic pigment in grapevine leaves (Vitis vinifera cv. "White Riesling”). Acta Horticulturae 754: 289-294. 